\title{
From Grothendieck to Naor: A Stroll Through the Metric Analysis of Banach Spaces
}

\author{
Gilles Godefroy (Institut de Mathématiques de Jussieu - Paris Rive Gauche, Paris, France)
}

In July 1954, Alexandre Grothendieck writes the introduction to his "cours relativement complet sur la théorie des espaces vectoriels topologiques, ou plus précisément sur la partie de la théorie qui peut être considérée comme le prolongement direct et l'aboutissement des idées de S. Banach", published in Sao Paulo under the title "espaces vectoriels topologiques". He states that Banach's theory has not really been surpassed in its essential results, which are the applications of the Baire and Hahn-Banach theorems. He mentions, though, a seminary on the "most recent developments in tensorial topological analysis", without specifying that these are due to him and that they represent a real surpassing of Banach's ideas and those of his school. In fact, these fundamental results had been published by Grothendieck the year before, also in Sao Paulo, in his famous Résumé [6]. Rather than being a "straightforward continuation of S. Banach's ideas", they offer a radically different point of view, even if we had to wait until 1968 for the importance of the Résumé to be internationally recognised, thanks to an article by Joram Lindenstrauss and Alexander Pelczynski. This article marks the beginning of the study of (metric, finite-dimensional, combinatorial) rigid structures in functional analysis, some examples of which can be seen below. A nonlinear component has recently been introduced in this field of research, which was motivated, in particular, by questions from computer science and where many young talents have obtained outstanding results over the past 15 years. Among this new generation, Assaf Naor plays a central role and the reader will notice that his work constitutes the thread of this note.

So why should we try to embed metric spaces into one Banach space or another? What importance can the numerical value of the Grothendieck constant possibly have? We do not ask these questions out of mere intellectual curiosity. They are indeed a way toward discoveries. Let us see how.

\section{The Ribe programme}

The theorem of M. I. Kadec (1967) states that any separable Banach space of infinite dimension is homeomorphic to the Hilbert space. This result was extended to the non-separable case in 1981 by $\mathrm{H}$. Torunczyk, who showed that two Banach spaces of the same density character are homeomorphic (where the density character of a space is the minimum of the set of cardinals of dense subsets). The topological theory of Banach spaces is thus trivial, in a sense. However, these first results fail to provide information when we consider applications that are not supposed to be linear but respect all or part of the metric structure, and can force the isomorphism; the classical theorem of Mazur-Ulam, for instance, states that any surjective isometry between Banach spaces is affine. In 1976, Martin Ribe published a very interesting theorem, which states that two uniformly homeomorphic Banach spaces have the same local structure, that is, the same subspaces of finite dimension up to an isomorphism constant. This means that if there exists a bijection $f$ between two Banach spaces $X$ and $Y$ such that $f$ and $f^{-1}$ are both uniformly continuous then there exists a constant $C>0$ such that for any subspace of finite dimension $E \subset X$, there exists a subspace $F \subset Y$ such that $F$ is $C$-isomorph to $E$ (so there exists a linear isomorphism $T$ from $E$ to $F$ such that $\|T\| .\left\|T^{-1}\right\| \leq C$ ) and conversely when $X$ and $Y$ are swapped. In simpler words, $X$ and $Y$ have the same subspaces of finite dimension. The local structure of a Banach space is thus a uniform invariant.

A quantitative form of the theorem of Ribe was given by Bourgain in 1987. To express it, we need the following notation, which will be used throughout this note. If $\left(M, d_{M}\right)$ and $\left(N, d_{N}\right)$ are two metric spaces and if $f: M \rightarrow N$ fulfils

$$
a d_{M}(x, y) \leq d_{N}(f(x), f(y)) \leq A d_{M}(x, y)
$$

for any pair $(x, y) \in M^{2}$, the quantity $A / a=D(f)$ is said to be the distortion of $f$. If there exists such a function $f$, we say that $M$ bi-Lipschitz embeds into $N$. In this case, we write

$$
c_{N}(M)=\inf \{D(f) ; f: M \rightarrow N \text { satisfies }(1)\}
$$

and, of course, $c_{N}(M)=+\infty$ if there is no such function $f$. In the particular case where $N=L_{p}$, endowed with its usual norm, we simply write

$$
c_{L_{p}}(M)=c_{p}(M) .
$$

The cases $p=2$ and $p=1$ will be particularly important. Using this notation, Bourgain's theorem of discretisation reads as follows. There exists an absolute constant $C>0$ such that if $\epsilon>0, Y$ is a normed space, $X$ is a normed space of dimension $n$ and $N$ is a $\delta$-lattice of $X$ with

$$
\delta<e^{-(n / \epsilon)^{C n}}
$$

then $c_{Y}(N) \geq(1-\epsilon) c_{Y}(X)$. We recall that $N$ is a $\delta$-lattice of $X$ if for any $x \in X$, one has $\inf \{\|x-y\| ; y \in N\} \leq \delta$. So, if a sufficiently fine lattice of $X$ embeds bi-Lipschitzly into $Y$, the same applies for the whole space $X$. The Ribe theorem follows, since a uniformly continuous map defined on a normed space becomes Lipschitz when restricted to a uniformly discrete lattice with a quantitative control. The theorem of discretisation suggests the existence of finite metric 
spaces, which represent an obstruction to a local property of Banach spaces.

Ribe's theorem gives a start to the Ribe programme, in the terminology of Joram Lindenstrauss and Jean Bourgain: given a local property $(p)$ of Banach spaces, find a property $(P)$ of metric spaces $M$ that coincides with $(p)$ when $M$ is a Banach space. The Ribe theorem states that this is possible, in principle, but will only be useful if the property $(P)$ is as simple and canonical as possible. The Ribe programme aims to transfer the properties of the structured field of Banach spaces to the larger class of metric spaces. It allows us to study metric spaces using our knowledge and intuition on the geometry of Banach spaces. Assad Naor is an eminent expert on this approach, which has turned out to be remarkably efficient when studying metric spaces, enabling us to find applications that might not have been discovered without the Ribe programme. The metric spaces hide rich structures that we are able to discover when we consider the right properties $(P)$ that derive from the local properties of Banach spaces.

Thus, the purpose of the Ribe programme is in particular the following. Given a local property $(p)$, find a good definition of a property $(P)$ of the metric spaces and, once it is defined, prove that when the metric space in question is a Banach space, $(P)$ reduces to $(p)$. If this approach is interesting for Banach spaces, it turns out to be the key to problems about metric spaces, which at first sight have no relation to normed spaces. Our main reference about the Ribe programme is [13] and the reader may also refer to the lecture by Keith Ball at the Bourbaki seminar [1].

A Banach space $X$ is said to be of type $p$, where $1 \leq p \leq 2$, if there exists $C>0$ such that

$$
2^{-n} \sum_{\epsilon_{i}= \pm 1}\left\|\sum_{i=1}^{n} \epsilon_{i} x_{i}\right\|_{X} \leq C\left(\sum_{i=1}^{n}\left\|x_{i}\right\|_{X}^{p}\right)^{1 / p}
$$

for all vectors $x_{1}, x_{2}, \ldots x_{n}$ in $X$. The triangular inequality yields that every space is of type 1 , so type $p$ appears as a strong triangular inequality (modulo a randomisation). The inequalities of Khintchine show that no Banach space can be of type $p>2$. On the other hand, a Banach space is of cotype $q$, with $2 \leq q<+\infty$, if one has

$$
\left(\sum_{i=1}^{n}\left\|x_{i}\right\|_{X}^{q}\right)^{1 / q} \leq C 2^{-n} \sum_{\epsilon_{i}= \pm 1}\left\|\sum_{i=1}^{n} \epsilon_{i} x_{i}\right\|_{X}
$$

Again, the inequalities of Khintchine show that the cotype of every space is bounded below by 2 . The spaces $l_{p}(1 \leq p<$ $+\infty)$ are of type $\inf (p, 2)$ and of cotype $\sup (p, 2)$. The theorem of Kwapien (1972) states that a Banach space $X$ is isomorphic to a Hilbert space if and only if $X$ is of type 2 and of cotype 2 .

From a geometric point of view, the definition of type can be seen as an inequality between the lengths of the diagonals of a parallelepiped and the lengths of their edges, which extends the Euclidean identity of the parallelogram. The nonlinear version, given by Per Enflo, is the following. A geometric cube of a metric space $M$ is a subset of $M$ indexed by $\{-1,1\}^{n}$. A diagonal is a pair $\left(x_{\epsilon}, x_{-\epsilon}\right)$ and an edge is a pair $\left(x_{\epsilon}, x_{\delta}\right)$, where $\epsilon$ and $\delta$ differ by only one coordinate. Then, $M$ has, by definition, metric type $p$ if one has

$$
2^{-n} \sum \text { diagonals } \leq C\left(2^{-n} \sum(\text { edges })^{p}\right)^{1 / p} .
$$

It is clear that a Banach space $X$ of metric type $p$ also has type $p$, and an inequality given by Gilles Pisier in 1986 shows (almost) the converse: if $X$ is of type $p$ then it is of metric type $p-\epsilon$ for all $\epsilon>0$. Gilles Pisier also showed, in 1973, that a Banach space $\mathrm{X}$ has a non-trivial type $p>1$ if and only if it does not contain uniformly the spaces $l_{1}^{n}$ (so, $\mathbf{R}^{n}$ equipped with the norm $\left.\|\cdot\|_{1}\right)$. In other words, $X$ only has the trivial type $p=1$ if and only if there exists $C>0$ such that for any $n$, there exists a subspace $E_{n}$ of $X$ that is $C$-isomorph to $l_{1}^{n}$. The corresponding metric result was shown in 1986 by $\mathrm{J}$. Bourgain, V. Milman and H. Wolfson: a metric space M has a type $p>1$ if and only if it does not contain uniformly biLipschitz copies of Hamming cubes $H_{n}=\left(\{-1,1\}^{n}, \|\right.$. $\left.\|_{1}\right)$. So, the uniform presence of Hamming cubes in a Banach space $X$ (i.e. $\left.\sup _{n}\left[c_{X}\left(H_{n}\right)\right]<\infty\right)$ is the metric obstruction to the nontrivial type for $X$.

It turns out to be difficult to find a good definition of the metric cotype but the problem was solved by Manor Mendel and Assaf Naor in 2008: a metric space $M$ is of cotype $q$ if there exists $C>0$ such that for every $n$, there exists $k$ such that for every function $f: \mathbb{Z}_{2 k}^{n} \rightarrow M$, one has

$$
\begin{aligned}
& \sum_{j=1}^{n} \sum_{x \in \mathbb{Z}_{2 k}^{n}} d_{M}(\left.f\left(x+k e_{j}\right), f(x)\right)^{q} \\
& \leq C k^{q} / 3^{n} \sum_{\epsilon \in\{-1,0,1\}^{n}} \sum_{x \in \mathbb{Z}_{2 k}^{n}} d_{M}\left(f(x+\epsilon, f(x))^{q},\right.
\end{aligned}
$$

where $e_{j}$ stands for the element of $\mathbb{Z}_{2 k}^{n}$ that takes the value 1 at position $\mathrm{j}$ and 0 elsewhere. With this definition, a Banach space $X$ is of cotype $q$ if and only if it has metric cotype $q$, leading to a metric analogue to the theorem of Bernard Maurey and Gilles Pisier (1976) saying that a Banach space is of cotype $q<+\infty$ if and only if it does not uniformly contain the spaces $l_{\infty}^{n}$. M. Mendel and A. Naor deduced from these considerations a very general dichotomy theorem.

Theorem 1. Let $\mathcal{F}$ be a family of metric spaces. Then, exactly one of the following assertions is true:

(i) For every finite metric space $F$ and every $\epsilon>0$, there exists $M \in \mathcal{F}$ such that $c_{M}(F) \leq 1+\epsilon$.

(ii) There exist $\alpha>0$ and $K>0$ such that for any integer $n$, there exists a metric space $M_{n}$ with $n$ points such that for all $N \in \mathcal{F}$, one has $c_{N}\left(M_{n}\right) \geq K(\log n)^{\alpha}$.

In other terms, if a family $\mathcal{F}$ is not quasi-isometrically universal for the finite metric spaces then spaces of cardinality $\mathrm{n}$ will show it with a distortion that grows, at least, like a power of $\log (n)$.

We have, thus, a convenient metric approach of type and cotype: let us remark on the latter that a Banach space $X$ has a trivial cotype $(+\infty)$ if and only if it contains bi-Lipschitzly all the locally finite metric spaces [2] with a distortion bounded from above by a universal constant. We recall that a Banach space $X$ is said to be super-reflexive when every space $Y$ with the same local structure as $X$ (that is, uniformly the same subspaces of finite dimension) is reflexive. The metric characterisation of super reflexivity was given by J. Bourgain in 1986: let $T_{n}^{k}$ be the $k$-regular tree of height $n$ equipped with the geodesic distance. Then, a Banach space $X$ is super reflexive if and only if, for every $k \geq 3$, one has

$$
\lim _{n \rightarrow+\infty} c_{X}\left(T_{n}^{k}\right)=+\infty \text {. }
$$


Bourgain also showed that, for a fixed $k$, the quantity $c_{2}\left(T_{n}^{k}\right)$ is of order $\sqrt{\log n}$. This leads naturally to the project of characterising the quantitative properties of the norms in metric terms, since the theorem of Enflo-Pisier states that a space is super reflexive if and only if it admits an equivalent norm that is uniformly convex and/or uniformly smooth, with the modulus of convexity and/or smoothness being controlled by a power of the parameter. J. R. Lee, M. Mendel, A. Naor and Y. Peres showed that the existence of a uniformly convex norm with a module in $\epsilon^{q}$ on $X$ was equivalent to the Markov type $q$ (a metric notion introduced by Keith Ball) but, so far, there has been no metric characterisation of the spaces for which there exists a uniformly smooth norm with module in $\eta^{p}$. We remark, along these lines, that if two Banach spaces $\mathrm{X}$ and $\mathrm{Y}$ contain Lipschitz-isomorphic lattices (which is the case when $\mathrm{X}$ and $\mathrm{Y}$ are uniformly homeomorphic) and if $\mathrm{X}$ has an asymptotically uniformly smooth norm with asymptotic modulus of power type $p$ then $Y$ will have, for all $\epsilon>0$, such a norm with an asymptotic modulus of power type $(p-\epsilon)$ and this $\epsilon>0$ disappears if $X$ and $Y$ are Lipschitz-isomorphic ([4] and Theorem 3.2 in [5]).

The most important result of the local theory of Banach spaces is undoubtedly the Dvoretzky theorem. Its role in the Ribe programme as well as the ideas it inspired are so important that we dedicate an entire chapter to it.

\section{The nonlinear versions of the Dvoretzky theorem}

In 1961, Aryeh Dvoretzky positively solved a conjecture formulated by Grothendieck in 1956, establishing the following fundamental theorem. Let $n$ be an integer and $\epsilon>0$. There exists an integer $N=N(n, \epsilon)$ such that if $X$ is a normed space of dimension $N$, there exists a linear map $T: l_{2}^{n} \rightarrow X$ such that $\|T\| .\left\|T^{-1}\right\|<1+\epsilon$ (where, of course, $T^{-1}$ is defined on the range of $T$ ). In other words, every normed space of sufficiently large dimension contains almost spherical sections. Works by T. Figiel, J. Lindenstrauss, V. Milman and Yehoram Gordon (1985) prove more precisely that there exists a universal constant $c$ such that if $0<\epsilon<1$ and $n \in \mathbb{N}$, one can use $N(n, \epsilon)=\exp \left[c n \epsilon^{-2}\right]$.

Together with the Ribe programme, the Dvoretzky theorem suggests the following conjecture. Given a finite metric space $M$, there exists a large subset $S$ of $M$ such that $c_{2}(S)$ is small; therefore, $S$ embeds into the Euclidian space with controlled distortion. The publications of Assaf Naor, some of them in collaboration with Manor Mendel, precisely establish this conjecture, which paved the way to many applications. Our main reference on this subject is [10]. This work proves the central role that is played by ultrametric spaces, which will be described below.

A metric space $M$ is said to be ultrametric if, for all $x, y$ and $z$ in $M$, one has $d(x, z) \leq \sup [d(x, y), d(y, z)]$. If a finite set $M$ is ultrametric, the relation $R$, defined by $x R y$ if $d(x, y)<\operatorname{diam}(M)$, is an equivalence relation. When applying this remark to every equivalence class (which is itself ultrametric) and iteratively, one can identify $M$ with the leaves of a tree equipped with the geodesic distance. It follows, in particular, that a finite ultrametric space and, more generally, a compact ultrametric space $M$ embeds isometrically into a
Hilbert space: $c_{2}(M)=1$. The main result of the work by Mendel and Naor is the theorem of the ultrametric skeleton [11], which reads as follows.

Theorem 2. For every $\epsilon>0$, there exists $c_{\epsilon} \in[1,+\infty)$ such that: for any compact metric space $M$ and any probability measure $\mu$ on $M$, there exists a compact subset $S$ of $M$ and a probability measure $v$ supported by $S$ such that $S$ embeds into an ultrametric space with distortion at most $9 / \epsilon$ and, for every $(x, r) \in M \times[0,+\infty)$, one has $v(B(x, r) \cap S) \leq\left(\mu\left(B\left(x, c_{\epsilon} r\right)\right)\right)^{1-\epsilon}$.

This ubiquity of ultrametric spaces, which was discovered through the Ribe programme, bears numerous consequences. Here is a first corollary.

Corollary 1. For any $\epsilon>0$ and any integer $n$, every finite metric space $M$ of cardinality $n$ contains a subset $S$ of cardinality at least $n^{1-\epsilon}$ embedded in an ultrametric space with distortion at most $(9 / \epsilon)$.

We now apply the theorem to a uniform probability $\mu$ on $M$ and to $r=0$. As $v$ is a probability on the subspace $S$, there exists $x \in S$ such that $v(\{x\}) \geq 1 /|S|$. But $v(\{x\}) \leq \mu(\{x\})^{1-\epsilon}=$ $1 / n^{1-\epsilon}$. Therefore, $|S| \geq n^{1-\epsilon}$.

Assaf Naor and Terence Tao have proven that in corollary 1 we can replace $(9 / \epsilon)$ by a bound $D(\epsilon)$ that tends toward 2 when $\epsilon$ tends toward 1. This is an aspect of the "phase transition at distortion 2", discovered by Y. Bartal, N. Linial, M. Mendel and A. Naor, of the maximum size of the approximately Euclidean subset of a metric space of cardinality $n$, which, when one crosses the distortion 2, passes from a power of $n$ to $\log (n)$. Assaf Naor and the above co-authors have also shown that there exist metric spaces of cardinality $n$ for which the corollary leads to an optimal result of existence of subsets that are embeddable into the Euclidean space. Therefore, up to a universal factor, the best way to find an approximately Euclidean subset is to find an approximately ultrametric one.

The corollary below uses a non-trivial probability measure $\mu$.

Corollary 2. For any $\epsilon \in(0,1)$ and any $\alpha \in(0,+\infty)$, every metric compact $M$ with its Hausdorff dimension greater than $\alpha$ contains a closed subset with a Hausdorff dimension bigger than $(1-\epsilon) \alpha$ that is embedded into an ultrametric space with distortion at most $(9 / \epsilon)$.

In order to deduce this corollary from Theorem 2, we use a Frostman measure $\mu$ on $M$, that is, a measure such that $\mu(B(x, r)) \leq C r^{\alpha}$ for all couples $(x, r)$, and then it can be easily proved that $S$ is convenient. The dimension of $S$ is, again, optimal, even for the approximately Euclidean subsets. A beautiful application of this corollary, given by T. Keleti, A. Mathe and O. Zindulka, is that if $K$ is a metric compact with Hausdorff dimension bigger than $n \in \mathbb{N}$, there exists a Lipschitz surjection of $K$ onto $[0,1]^{n}$.

Theorem 2 is linked to the theorem of majorising measures by Michel Talagrand [16]. We recall that if $X$ is a metric space and $\mathcal{P}_{X}$ the set of probability measures on $X$, the functional $\gamma_{2}(X)$ of Fernique-Talagrand is defined by the formula

$$
\gamma_{2}(X)=\inf _{\mu \in \mathcal{P}_{X}} \sup _{x \in X} \int_{0}^{+\infty} \sqrt{-\log (\mu(B(x, r)))} d r .
$$

An important step in the proof is to construct in every finite 
metric space $X$ a subset $S$ that embeds (with an absolute upper bound on the distortion) into an ultrametric space, such that one has $\gamma_{2}(X) \leq C \gamma_{2}(S)$. It turns out that Theorem 2 allows us to prove the theorem of majorising measures, by describing it as the result of an integration on $r$ of pointwise estimates. We can see the link between the two theorems through the role of the trees with orthogonal branches, which represent the Gaussian processes with independent increments and also allow the Euclidean embedding of ultrametric spaces.

Corollary 1 has, in addition, applications to theoretical computer science. This is not surprising, since an essentially Euclidean set constitutes a field where linear algebra and the numerous algorithms it contains can display all their power. Besides, we know that Naor, who is today at the Department of Mathematics at Princeton University, was formerly a member of Microsoft Research. Let us turn to the approximate distance oracle. A metric space with $n$ points is completely determined by the distances between points, that is, by $n(n-1) / 2$ pieces of data. However, the triangular inequality shows that those data are not independent. There exists a certain redundancy, which invites us to search for an essential subset of distances that will allow the estimation of all the others with a given precision. Corollary 1 allowed M. Mendel and A. Naor to prove the existence of a "constant query time oracle" for the approximate distances as follows.

Corollary 3. Let $D$ be strictly greater than 1. Any metric space $M$ of $n$ points can be preprocessed in a time $O\left(n^{2}\right)$, in a way so as to stock a number $O\left(n^{1+O(1 / D)}\right)$ of data such that, given $(x, y) \in M^{2}$, one obtains, in a uniformly bounded time, a number $E(x, y)$ that satisfies $d(x, y) \leq E(x, y) \leq \operatorname{Dd}(x, y)$.

The importance of this result (which was improved quantitatively in 2014 by S. Chechik, using similar methods) lies in the fact that the query time is bounded by a universal constant and therefore depends neither on $D$ nor on $n$. At the price of a certain distortion $D$, it is possible to control both the query time and the size of the data.

\section{Grothendieck inequality and combinatorial optimisation}

In his article from 1953 that founds the metric theory of tensor products and the modern theory of continuous linear operators between Banach spaces [6], Alexandre Grothendieck showed the following inequality. There exists a constant $K_{G}$ such that, if $S_{H}$ refers to the unit sphere of the Hilbert space (usually denoted $H$ ), one has, for all integers $n$ and $m$ and any real matrix $\left(a_{i j}\right)$,

$$
\begin{gathered}
\sup \left\{\sum_{i=1}^{m} \sum_{j=1}^{n} a_{i j}\left\langle x_{i}, y_{j}\right\rangle ;\left(x_{i}\right),\left(y_{j}\right) \subset S_{H}\right\} \\
\leq K_{G} \sup \left\{\sum_{i=1}^{m} \sum_{j=1}^{n} a_{i j} \epsilon_{i} \delta_{j} ; \epsilon_{i}= \pm 1, \delta_{j}= \pm 1\right\} .
\end{gathered}
$$

In other words, if we replace the Hilbert space on the left by the set of real numbers, the supremum is of the same order. There exists a complex version of this inequality, which differs from the real version only by the value of the constant $K_{G}$.
In his seminal article, Grothendieck applies this inequality to the linear operators, proving, among many other results, that every operator $T$ from $L_{\infty}$ to $L_{1}$ factors through a Hilbert space, i.e. that there exist operators $A: L_{\infty} \rightarrow H$ and $B$ : $H \rightarrow L_{1}$ such that $T=B A$. An essential reference regarding applications of the Grothendieck inequality is Gilles Pisier's article from 2012 [15].

The link between the Grothendieck inequality and combinatorial optimisation, for which article [7] is our main reference, is established by the interpretation of inequality (2) as a vectorial relaxation of the estimate of a supremum. Given a sufficiently regular compact convex set $K$ of semidefinite positive symmetric matrices $k \times k$ and a matrix $\left(c_{i j}\right)$, one can, in polynomial time, compute the maximum of the quantity

$$
\sum_{i=1}^{k} \sum_{j=1}^{k} c_{i j} x_{i j}
$$

on all matrices $\left(x_{i j}\right)$ belonging to $K$. The computation of the left side of (2) is an example of this technique (called semidefinite programming, our reference being [14]) and can therefore be done in polynomial time in $k$ with arbitrary precision. This is consequently the case for the right side of (2), up to the constant $K_{G}$, of course. The right side is the norm of the matrix $\left(a_{i j}\right)$, considered as a linear map from $l_{\infty}^{n}$ to $l_{1}^{m}$.

Following Noga Alon and Assaf Naor in their discovery, we investigate the link between inequality (2) and the estimation of the cut norm of an $m \times n$ matrix $A=\left(a_{i j}\right)$, defined by

$$
\|A\|_{c u t}=\max _{S, T}\left|\sum_{i \in S, j \in T} a_{i j}\right|,
$$

where the maximum is taken over the subsets $S \subset\{1, \ldots, m\}$ and $T \subset\{1, \ldots n\}$. Let $B$ be the matrix of size $(m+1) \times(n+1)$ obtained by attaching to $A$ a column and a line, in such a way that all lines and all columns of $B$ are of zero sum. A quite simple direct calculation proves that

$$
\|A\|_{\text {cut }}=\frac{1}{4}\|B\|_{\infty \rightarrow 1} .
$$

The semidefinite programming allows one to calculate the quantity $\|B\|_{\infty \rightarrow 1}$ in polynomial time, up to the constant $K_{G}$. The same holds for the norm $\|A\|_{c u t}$ and there exists, accordingly, an algorithm of polynomial time that computes a quantity $\alpha(A)$ such that

$$
\|A\|_{\text {cut }} \leq \alpha(A) \leq C\|A\|_{c u t},
$$

with $C=K_{G}$. However, unless $P=N P$, such an algorithm does not exist if $C<13 / 12$, and P. Raghavendra and D. Steurer have proven that modulo the combinatorial conjecture named (UGC) (unique games conjecture), the Grothendieck constant is optimal for the existence of an algorithm of polynomial time that provides $\alpha(A)$ satisfying (3). We will return to the numerical value of the Grothendieck constant below.

The regularity lemma by Szemeredi states informally that every combinatorial graph $G$ (a finite set $V$ of vertices pairwise linked or not by edges forming a set $E \subset V^{2}$ ) can be partitioned into a controlled number of subsets that interact pseudo-randomly. More precisely, if $X$ and $Y$ are disjoint subsets of $V$, one denotes by $e(X, Y)=|(X \times Y) \cap E|$ the cardinal of the set of edges that join $X$ and $Y$. If $\epsilon>0$ and $\delta>0$, we 
say that the pair $(X, Y)$ is $(\epsilon, \delta)$-regular if, once $S \subset X$ and $T \subset Y$ satisfy $|S| \geq \delta|X|$ and $|T| \geq \delta|Y|$, one has

$$
\left|\frac{e(S, T)}{|S| .|T|}-\frac{e(X, Y)}{|X| .|Y|}\right| \leq \epsilon .
$$

This quasi-uniformity of the quantities $e(S, T) /|S| .|T|$ means that the pair $(X, Y)$ is pseudo-random, that is to say, close to a bipartite graph where every pair $(x, y) \in X \times Y$ is joined independently by an edge with probability $e(X, Y) /|X| .|Y|$. In order to construct Szemeredi partitions in polynomial time, the important step is to determine in polynomial time if a pair $(X, Y)$ is close to being $(\epsilon, \delta)$-regular. For this purpose, $\mathrm{N}$. Alon and A. Naor consider, given two disjoint subsets $X$ and $Y$ of cardinality $n$ of $V$, the matrix $A=\left(a_{x y}\right)$ indexed by $(X \times Y)$, such that

$$
a_{x y}=1-\frac{e(X, Y)}{|X| .|Y|}
$$

if $(x, y) \in E$ and

$$
a_{x y}=-\frac{e(X, Y)}{|X| .|Y|}
$$

otherwise. Therefore, the matrix $A$ is the difference of the adjacency matrix of the graph $G$ restricted to $X \times Y$ and a matrix whose entries are the probabilities that two vertices of $X$ and $Y$ are connected. It can easily be verified that if $(X, Y)$ is not $(\epsilon, \delta)$-regular then $\|A\|_{c u t} \geq \epsilon \delta^{2} n^{2}$. The algorithm for the computation of the cut norm (up to $K_{G}$ ) in polynomial time allows one to decide in polynomial time if $(X, Y)$ is $(\epsilon, \delta)$-regular or to find a pair of parts of $X$ and $Y$ that prove it is not (for other values of $\epsilon$ and $\delta$ ). This also requires a polynomial time for the procedure of rounding, which we describe below.

Our goal is to find the choices of signs $\left(\epsilon_{i}\right)$ and $\left(\delta_{j}\right)$, whose existence is guaranteed by inequality (2) and which fulfil this inequality. To achieve this, we will apply a method developed by Jean-Louis Krivine in 1977. Let $f$ and $g$ be two measurable odd functions from $\mathbb{R}^{k}$ to $\{-1,1\}$. Let $G_{1}$ and $G_{2}$ be two independent arbitrary Gaussian vectors in $\mathbb{R}^{k}$. For $t \in(-1,1)$, we define

$$
H_{f, g}(t)=\mathbb{E}\left[f\left(\frac{1}{\sqrt{2}} G_{1}\right) g\left(\frac{t}{\sqrt{2}} G_{1}+\frac{\sqrt{1-t^{2}}}{\sqrt{2}} G_{2}\right)\right] .
$$

Under a simple condition, an analytic method allows one to determine a scalar $c(f, g)$ such that if $\left(x_{i}\right),\left(y_{j}\right)$ are vectors of norm 1 in (2), there exist unit vectors $\left(u_{i}\right),\left(v_{j}\right)$ in $\mathbb{R}^{m+n}$ such that for all $1 \leq i \leq m$ and $1 \leq j \leq n$, one has

$$
\left\langle u_{i}, v_{j}\right\rangle=H_{f, g}^{-1}\left(c(f, g)\left\langle x_{i}, y_{j}\right\rangle\right) \text {. }
$$

Now, let $G$ be a random $k \times(m+n)$ matrix whose coefficients are independent standard Gaussian variables. If one sets

$$
\epsilon_{i}=f\left(\frac{1}{\sqrt{2}} G u_{i}\right), \delta_{j}=g\left(\frac{1}{\sqrt{2}} G v_{j}\right),
$$

we find

$$
\mathbb{E}\left[\sum_{i=1}^{m} \sum_{j=1}^{n} a_{i j} \epsilon_{i} \delta_{j}\right]=c(f, g) \sum_{i=1}^{m} \sum_{j=1}^{n} a_{i j}\left\langle x_{i}, y_{j}\right\rangle .
$$

This identity produces the rounding algorithm in polynomial time to produce a conveniant choice of signs for (2), also applicable to the $(\epsilon, \delta)$-regular couples of Szemeredi. Besides that, it allows one to bound $K_{G}$, since it proves that for every pair $(f, g)$, one has $c(f, g)^{-1} \geq K_{G}$. Using $f=g=$ $\operatorname{sign}(x)$, Jean-Louis Krivine has shown by this method that
$K_{G} \leq \pi /(2 \log (1+\sqrt{2}))$, and this is the best possible result when considering the odd functions defined on $\mathbb{R}$. However, M. Braverman, M. Makarychev, K. Makarychev and Assaf Naor proved in 2011 that if $f=g$ corresponds to the partition of the plane on both sides of the graph of the polynomial $y=c\left(x^{5}-10 x^{3}+15 x\right)$ with a well chosen $c>0$, the resulting estimation is better than the one given by Krivine. This solved a problem that had been open since 1977. So, the current estimates for the Grothendieck constant are:

$$
\frac{\pi}{2} e^{\eta_{0}^{2}}=1.676 \ldots \leq K_{G}<\pi /(2 \log (1+\sqrt{2}))=1.782 \ldots,
$$

where $\eta_{0}=0.25573 \ldots$ is the only solution to the equation

$$
1-2 \sqrt{\frac{2}{\pi}} \int_{0}^{\eta} e^{-z^{2} / 2} d z=\frac{2}{\pi} e^{-\eta^{2}}
$$

This lower bound was obtained in 1991 by J. A. Reeds in an unpublished work.

M. Braverman, M. Makarychev, K. Makarychev and Assaf Naor have conjectured that the best Krivine scheme in dimension 2 corresponds to two distinct odd partitions $f$ and $g$, where $f$ is the following "tiger fur":

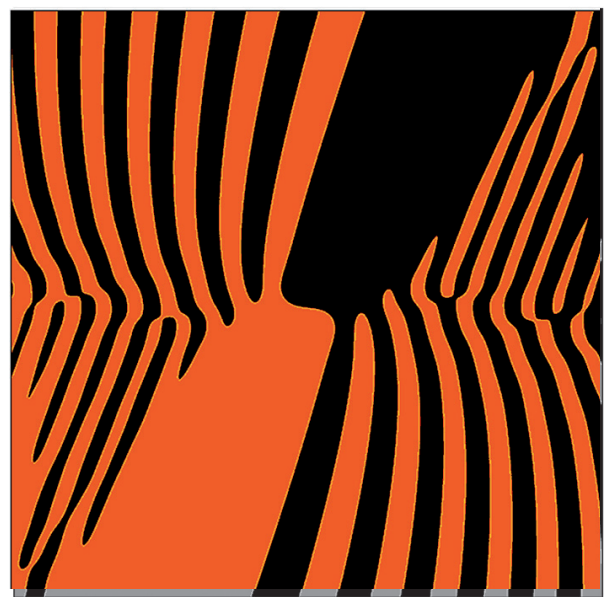

It is likely that Krivine schemes in dimension $k \geq 3$ will lead to finer bounds on the Grothendieck constant.

Let us follow the thoughts of Assaf Naor and his coauthors on the combinatorial aspects of inequality (2). Let $G$ be a graph with $n$ vertices denoted by $\{1, \ldots n\}$ and $E \subset$ $\{1, \ldots n\}^{2}$ the set of its edges. The Grothendieck constant of $G$, denoted by $K(G)$, is the smallest constant $K$ such that

$$
\sup _{\left(x_{i}\right) \subset S_{H}} \sum_{(i, j) \in E} a_{i j}\left\langle x_{i}, x_{j}\right\rangle \leq K \sup _{\epsilon_{i}= \pm 1} \sum_{(i, j) \in E} a_{i j} \epsilon_{i} \epsilon_{j}
$$

holds true for any real matrix $A=\left(a_{i j}\right)$. The Grothendieck inequality (2) is the particular case of (4) that corresponds to the bipartite graphs (i.e. of chromatic number 2) and, as a consequence,

$$
K_{G}=\sup _{n}\{K(G) ; G \text { bipartite graph with } n \text { vertices }\} .
$$

Additionally, if $K_{n}$ stands for the complete graph with $n$ vertices, the corresponding Grothendieck constant is of order $\log (n)$. The Grothendieck constant of a graph $G$ is clearly related to the combinatorics of $G$ and has, as such, its own interest. On the other hand, the right term in (4) is relevant to statistical physics: if $G$ weighted by the matrix $A$ represents the possible interactions of $n$ particles affected by a spin $\epsilon_{i}= \pm 1$ 
then the total energy generated by these particles in the system in the Ising model of the spin glass is $E=-\left(\sum_{(i, j) \in E} a_{i j} \epsilon_{i} \epsilon_{j}\right)$. A configuration of the spins $\left(\epsilon_{i}\right) \in\{-1,1\}^{n}$ represents a ground state if it minimises the total energy.

So, finding a ground state of the spin glass corresponds to maximising the right term in (4). It is known that this can be done in polynomial time for the planar graphs and that it is an NP-complete problem if $G$ is a grid in dimension 3. However, as the quantity on the left side in (4) is related to semidefinite programming, the right side can be calculated in polynomial time up to a factor $K(G)$.

We would like to close this section by mentioning the work of M. Charikar and A. Wirth on the case of the graphs $G=(V, E)$ with their edges weighted by 1 or -1 if the vertices are considered to be "similar" or "different", the absence of an edge meaning that no judgement is given regarding the similarity of the corresponding vertices. The problem to be solved is to split the graph $G$ in a manner that maximises the number of similarities between the members of the same subset of the partition as well as the number of differences between members of different subsets. The bottleneck of the construction is to obtain a partition in two subsets, which again means maximising the right side in (4) and consequently requires the same techniques.

\section{$4 \quad$ Extensions of Lipschitz functions}

Let $M$ be a metric space and $S$ a non-empty subset of $M$. A formula given by MacShane (1934) proves that any Lipschitz function $f: S \rightarrow \mathbb{R}$ extends to a function with the same Lipschitz constant on $M$. In order to obtain such an extension, all we need to do is to set

$$
\bar{f}(m)=\inf \{f(s)+\operatorname{Lip}(f) d(m, s) ; s \in S\} .
$$

As we know, a function $f: M \rightarrow N$ between two metric spaces is said to be Lipschitz if there exists $C>0$ such that $d_{N}(f(x), f(y)) \leq C d_{M}(x, y)$ for all $(x, y) \in M^{2}$, and the minimum of the constants $C$ for which these inequalities are fulfilled is called the Lipschitz constant of $f$ and is denoted $\operatorname{Lip}(f)$.

MacShane's formula is a very useful tool but has two shortcomings: firstly, it is not linear in $f$ and secondly, it essentially uses the order structure on $\mathbb{R}$. It is therefore not directly usable for the similar question of the extension of Lipschitz functions with values in a Banach space. A positive result (contemporary to MacShane's formula) is Kirszbraun's theorem, which states that if $S$ is a subset of a Hilbert space $H$, every Lipschitz map from $S$ to $H$ can be extended with the same Lipschitz constant to $H$. However, Joram Lindenstrauss proved in 1963 that this result cannot be extended to Banach spaces, even if we allow extensions with arbitrary Lipschitz constants. This more general frame provides specific problems, since a "point by point" extension technique and Zorn's lemma will not lead to a result if the Lipschitz constant explodes during construction. In his $\mathrm{PhD}$ (directed by J. Lindenstrauss, himself a student of Dvoretzky), Assaf Naor showed, in particular, that a Lipschitz map from a subset $S$ of a Hilbert space $H$ to a Banach space $X$ can't generally extend Lipschitzly to $H$, even if, for instance, $X=L_{4}$. The Kirszbraun theorem is, thus, essentially optimal. How- ever, on the positive side, A. Naor, Y. Peres, O. Schramm and S. Sheffield established the nonlinear version of a classical result by Bernard Maurey: every Lipschitz function of a subset $S$ of a 2-uniformly smooth space $X$ in a 2-uniformly convex space $Y$ extends Lipschitzly to the space $X$.

This theorem is only one of the results on Lipschitz extensions obtained by Assaf Naor. Our reference for the rest of this section will be his article from 2005 with James R. Lee [9]. One of the difficulties we need to overcome in relation to the extensions is the transition from a local to a global extension. To achieve this, one of the ideas of Lee and Naor was to use partitions of unity that are subordinated to a random covering, and to obtain the desired regularity by taking the average. This analytic approach will be combined with some particular decompositions taken from theoretical computer science and adapted here to infinite sets, respecting the conditions for measurability.

In the following, we would like to present more precisely the least technical concept from this approach: the gentle partitions of unity. If $M$ is a metric space, $S$ a closed subset of $M$ and $(\Omega, \mu)$ a measured space, and if $K>0$, a function $\Psi: \Omega \times M \rightarrow(0,+\infty)$ is a $K$-gentle partition of unity relative to $S$ if the following applies:

(i) For every $x \in M \backslash S$, the function $\omega \rightarrow \Psi(\omega, x)$ is measurable and $\int_{\Omega} \Psi(\omega, x) d \mu(\omega)=1$.

(ii) If $x \in S$, we have $\Psi(\omega, x)=0$ for every $\omega \in \Omega$.

(iii) There exists a Borel function $\gamma: \Omega \rightarrow S$ such that, for every $(x, y) \in M^{2}$,

$$
\int_{\Omega} d(\gamma(\omega), x) \cdot|\Psi(\omega, x)-\Psi(\omega, y)| d \mu(\omega) \leq K d(x, y) .
$$

If $f$ is a Lipschitz function defined on $S$ on values in a Banach space $Z$, we set, for any $x \in M \backslash S$,

$$
\bar{f}(x)=\int_{\Omega} f(\gamma(\omega)) \Psi(\omega, x) d \mu(\omega)
$$

and $\bar{f}(x)=f(x)$ if $x \in S$. It can easily be seen that $\bar{f}$, which of course extends $f$, is Lipschitz and fulfils $\operatorname{Lip}(\bar{f}) \leq 3 K \operatorname{Lip}(f)$. Note that the extension $\bar{f}$ obtained by this formula depends linearly upon $f$.

The problem is now to establish the existence of gentle partitions. Lee and Naor prove that, referring to the subset $S$, being doubling is a sufficient condition. We know that a metric space $M$ is said to be doubling, with doubling constant $\lambda(M)$, if any ball of $M$ is contained in the union of $\lambda(M)$ balls of half radius. They then prove the following.

Theorem 3. There exists a universal constant $C>0$ such that if $M$ is a doubling metric space, of doubling constant $\lambda(M), Y$ a metric space that contains $M$ isometrically and $Z$ a Banach space then every Lipschitz function $f: M \rightarrow Z$ extends to a Lipschitz function $\bar{f}: Y \rightarrow Z$ such that $\operatorname{Lip}(\bar{f}) \leq$ $C \log (\lambda(M)) \operatorname{Lip}(f)$.

This theorem generalises and unifies previous results of W. B. Johnson, J. Lindenstrauss and G. Schechtman. If $M$ is a finite metric space of cardinality $n$ then $\log (\lambda(M))=$ $O(\log (n))$. Furthermore, if $M$ is a subset of a space $\mathbb{R}^{d}$ then $\log (\lambda(M))=O(d)$. Note that, for spaces of cardinality $n$, Lee and Naor prove that there exists an extension that satisfies $\operatorname{Lip}(\bar{f}) \leq C(\log (n) / \log (\log (n))) \operatorname{Lip}(f)$. 
James R. Lee and Assaf Naor also show that, in Theorem 3, one can replace the doubling space $M$ by a subset of a Riemann surface of genus $g$, and then one has $\operatorname{Lip}(\bar{f}) \leq$ $C(g+1) \operatorname{Lip}(f)$. On the other hand, recall that a graph $G$ excludes a graph $H$ if one cannot find $H$ based on $G$ by iterating the following operations: removing an edge or collapsing it by identifying the two vertices joined by that edge. Lee and Naor then prove that if a graph $G$ excludes the complete graph of $n$ vertices $K_{n}$ then $G=M$ satisfies the conclusion of Theorem 3 with $\operatorname{Lip}(\bar{f}) \leq C n^{2} \operatorname{Lip}(f)$. Thus, the trees (that is, the graphs that exclude $K_{3}$ ) uniformly satisfy Theorem 3, which is a theorem due to Jiri Matoušek. Furthermore, the planar graphs (which exclude $K_{5}$ ) satisfy the same conclusion, up to a constant. Interestingly, Theorem 3 also allows one to prove that if $M$ is a doubling metric space, the free space $\mathcal{F}(M)$ on $M$ has Grothendieck's bounded approximation property [8].

\section{$5 \quad$ Lipschitz embeddings}

We begin this section with a closer look at the doubling metric spaces $M$, for which exists a constant $\lambda(M)$ such that any ball in $M$ is contained in the union of $\lambda(M)$ balls of half radius. Patrice Assouad obtained, in 1983, the following noteworthy result. Let $(M, d)$ be a doubling space. For any $\alpha \in(0,1)$, one denotes by $M_{\alpha}$ the space $M$ equipped with the distance $d^{\alpha}$ defined by $d^{\alpha}(x, y)=[d(x, y)]^{\alpha}$. Then, there exists $N(\alpha)$ such that the space $M_{\alpha}$ embeds bi-Lipschitzly into $l_{2}^{N(\alpha)}$, i.e. into $\mathbb{R}^{N(\alpha)}$ endowed with the Euclidean distance. The operation that consists of replacing a distance $d$ by $d^{\alpha}$ with $0<\alpha<1$ is called snowflaking. Assouad's theorem was improved in 2012 by Assaf Naor and Ofer Neiman as follows. If $K>0$ and $\epsilon \in(0,1 / 2)$, there exist $N=N(K)$ and $D=D(K, \epsilon)$ such that if $M$ is a $K$-doubling metric space then $M_{1-\epsilon}$ embeds biLipschitzly into $l_{2}^{N(K)}$ with distortion $D(K, \epsilon)$. Compared to the original result by Assouad, the improvement is that the dimension $N$ does not depend on $\epsilon$ anymore.

Another groundbreaking contribution to the theory of Lipschitz embeddings was made by Jean Bourgain in 1986: any finite metric space of cardinality $|M|$ embeds into the Hilbert space with a distortion $D=O(\log (|M|))$. It is convenient to compare this result with the theorem by Fritz John, which states that the Banach-Mazur distance of a normed space of dimension $n$ to $l_{2}^{n}$ is bounded from above by $\sqrt{n}$. The example of the cubes $M=\{0,1\}^{k}$ might suggest that the metric analogue of the dimension of a finite space $M$ is $\log (|M|)$, and the expected estimation would be $O(\sqrt{\log (|M|)}$. The upper bound given by Bourgain is, however, optimal, which follows, in particular, from an essentially nonlinear phenomenon: the existence of expanding graphs. Assaf Naor and his collaborators have given further examples from harmonic analysis, as well as a noteworthy link to Assouad's theorem: any finite metric space $M$ of doubling constant $\lambda(M)$ embeds into the Hilbert space with a distortion $D=O(\sqrt{\log (\lambda(M)) \log (|M|)}$. Bourgain's theorem then follows from the trivial bound $\lambda(M) \leq$ $|M|$. There is an important lemma by Bill Johnson and Joram Lindenstrauss on dimension reduction that we would like to mention: if $M$ is a finite subset of the Hilbert space $H$ and $\epsilon>0$, there exists a bi-Lipschitz map $f: M \rightarrow l_{2}^{n}$ (which is, in fact, the restriction of a linear map of $H$ in $H$ ) of distortion $D(f)<1+\epsilon$, with $\left.n=O\left(\log (|M|) / \epsilon^{2}\right)\right)$. In a joint work published in 2010, Bill Johnson and Assaf Naor showed that a Banach space $X$ that fulfils the conclusion of this lemma is very close to a Hilbert space, without necessarily being itself Hilbertian. In particular, $L^{p}$ only fulfils the conclusion if $p=2$.

Following Assaf Naor's contribution to the international congress in Hyderabad in 2010 [12], we now examine the connections between these embedding theorems, the Heisenberg group and the algorithms for solving the Sparsest Cut problem. These connections are relevant to the geometric theory of groups, for which our reference is Etienne Ghys' Bourbaki lecture [3] and, of course, the work of M. Gromov. We denote by $\mathbb{H}$ the Heisenberg group considered as the space $\mathbb{R}^{3}$ endowed with the structure of a non-commutative group described by

$$
(a, b, c) \cdot\left(a^{\prime}, b^{\prime}, c^{\prime}\right)=\left(a+a^{\prime}, b+b^{\prime}, c+c^{\prime}+a b^{\prime}-b a^{\prime}\right) .
$$

We denote by $\mathbb{H}(\mathbb{Z})$ its discrete subgroup $\mathbb{Z}^{3}$. If we equip $\mathbb{H}(\mathbb{Z})$ with the word distance related to a finite family of generators (equivalent to the Carnot-Carathéodory distance), we obtain a doubling metric space. It follows from the theorem of differentiability by Pierre Pansu that this doubling space does not bi-Lipschitz embed into the Hilbert space, which leads to the necessity of snowflaking in Assouad's theorem. More generally, Pansu's theorem implies that $\mathbb{H}(\mathbb{Z})$ does not embed into $L_{p}$ with $1<p<+\infty$ but is not applicable to possible embeddings in $L_{1}$.

A metric space $(M, d)$ is said to be of negative type if its snowflaking $M_{1 / 2}=\left(M, d^{1 / 2}\right)$ embeds isometrically into the Hilbert space. The space $L_{1}$ is of negative type. The group $\mathbb{H}(\mathbb{Z})$, equipped with an equivalent distance, is also of negative type. We now describe the relationship between these particular metric spaces and the Sparsest Cut problem.

Let $C$ and $D$ be two symmetric functions $\{1, \ldots n\} \times$ $\{1, \ldots n\} \rightarrow(0,+\infty)$, named "capacity" and "demand". If $S$ is a non-empty subset of $\{1, \ldots n\}$, we set

$$
\Phi(S)=\frac{\sum_{i=1}^{n} \sum_{j=1}^{n} C(i, j)\left|\mathbf{1}_{S}(i)-\mathbf{1}_{S}(j)\right|}{\sum_{i=1}^{n} \sum_{j=1}^{n} D(i, j)\left|\mathbf{1}_{S}(i)-\mathbf{1}_{S}(j)\right|} .
$$

We now seek to estimate the quantity $\Phi^{*}(C, D)=\min _{S} \Phi(S)$, which is the smallest possible ratio of capacity/demand for cuts between $S$ and its complement, hence the name Sparsest Cut. The particular case where $C(i, j)=1$ if $i$ and $j$ are joined by an edge and $C(i, j)=0$ otherwise, and where $D=1$, is the problem of isoperimetry of $G$ : find a subset $S$ such that the relation of the cardinal of its border (i.e. the edges between $S$ and $V \backslash S$ ) to its cardinal is the smallest possible. It is known that the computation of $\Phi^{*}(C, D)$ is $N P$-complete. Under the combinatorial conjecture (UGC), this is even the case for the estimation of $\Phi^{*}(C, D)$ up to a constant: we cannot expect to find a "Grothendieck constant" in this case as we did in Section 3. However, a similar approach is used in Naor's article.

A first step is to use an extreme ray argument to prove the equation

$$
\Phi^{*}(C, D)=\min _{\left(f_{i}\right) \subset L_{1}} \frac{\sum_{i=1}^{n} \sum_{j=1}^{n} C(i, j)\left\|f_{i}-f_{j}\right\|_{1}}{\sum_{i=1}^{n} \sum_{j=1}^{n} D(i, j)\left\|f_{i}-f_{j}\right\|_{1}} .
$$

We can now consider the minimisation problem in the larger space of all metrics on $\{1, \ldots, n\}$, which leads to a problem of linear programming, solvable in polynomial time. But according to Bourgain's theorem, a minimising distance will be 
at a distance controlled by $\log (n)$ of a Euclidean distance, which is embeddable in $L_{1}$ (which isometrically contains $l_{2}$ via a sequence of independent Gaussian variables). We then deduce from (5) that we have solved the Sparsest Cut problem in polynomial time, up to a factor $\log (n)$. A look into the proof of Bourgain's theorem also shows us a way of obtaining a set $S$ that realises a small cut.

However, there is an even better way, which is to use the fact that the metric of $L_{1}$ is of negative type. Let $M(C, D)$ be defined as the minimum of the quantity $\sum_{i=1}^{n} \sum_{j=1}^{n} C(i, j) d_{i j}$ under the constraint that $\sum_{i=1}^{n} \sum_{j=1}^{n} D(i, j) d_{i j}=1$ and $d_{i j}$ is a metric of negative type. As in Section 3, we can calculate in polynomial time the quantity $M(C, D)$ by semidefinite programming. It is clear that $M(C, D) \leq \Phi^{*}(C, D)$. Equation (5) additionally shows that $\Phi^{*}(C, D) \leq C_{n} M(C, D)$, where we set

$$
C_{n}=\sup \left\{c_{1}(\{1, \ldots, n\}, d) ; d \text { a metric of negative type }\right\}
$$

and this inequality is exactly optimal. Thus, in order to approximately solve the Sparsest Cut problem in polynomial time, we are led to examine the embedding of finite metric spaces in $L_{1}$. Assaf Naor proves (together with S. Arora and $\mathrm{J}$. R. Lee) that $c_{1}(M) \leq c_{2}(M)=O\left(\log (|M|)^{1 / 2+o(1)}\right)$ if $M$ is of negative type but also (together with J. Cheeger and B. Kleiner) that $c_{1}\left(M_{n}\right) \geq(\log (n))^{c}$ for a certain $c>0$ if $M_{n}$ is the subset $\{1, \ldots, n\}^{3}$ of $\mathbb{H}(\mathbb{Z})$. The quantity $o(1)$ that appears in the first result is an artefact of the proof, controlled by $(\log \log \log n) /(\log \log n)$, and it is likely it can be eliminated; this is at least possible in the particular case of the isoperimetric problem. Work (since 2014) of Vincent Lafforgue and Assaf Naor suggested the existence of finite sets such that the constant $c$ in the second result should be the optimal value $1 / 2$, and this was indeed shown by A. Naor and R. Young in 2017, using the 5-dimensional Heisenberg group. Hence, the bi-Lipschitz embedding of metric spaces of negative type in $L_{1}$ gives a solution to the Sparsest Cut problem in polynomial time, up to a proportion $O(\sqrt{\log n})$.

We now come to the end of our portrait of recent work on metric theory of normed spaces but would like to invite the reader to consult the original articles. To conclude, let us frame the current approach in its historical context. In the past century, existence theorems in analysis have mostly been obtained by more or less constructive topological methods, relying, for instance, on compactness or completeness, or on fine combinatorial results. After that, probabilistic methods have been powerful tools, which have established, by the use of random methods, the existence of numerous objects that were not provided by explicit construction. The spectacular explosion of our computational power invites us no longer to seek non-constructive existence theorems that are unsatisfactory for those who need to apply them. We are now able to determine if and how an algorithm can provide the mathematical object for which we search, in an optimal or almost optimal manner. All of the highlighted contributions clearly belong to that third generation. Let us keep up with recent progress: the 21 st century has only just begun.

\section{Bibliography}

[1] K. Ball, The Ribe Programme, Séminaire Bourbaki, Vol. 2011-2012, Exposé 1047, Astérisque 352 (2013).
[2] F. Baudier, G. Lancien, Embeddings of locally finite metric spaces into Banach spaces, Proc. AMS 136 (2008), 10291033.

[3] E. Ghys, Les groupes hyperboliques, Séminaire Bourbaki, Vol. 1989-90, Exposé 722, Astérisque 189-190 (1990).

[4] G. Godefroy, N. J. Kalton, G. Lancien, Szlenk indices and uniform homeomorphisms, Trans. AMS, 353 (2001), 3895-3918.

[5] G. Godefroy, G. Lancien, V. Zizler, The non-linear geometry of Banach spaces after Nigel Kalton, Rocky Mountain J. of Maths 44 (2014), 1529-1583.

[6] A. Grothendieck, Résumé de la théorie métrique des produits tensoriels topologiques, Bull. Soc. Mat. Sao-Paulo 8 (1953), $1-79$.

[7] S. Khot, A. Naor, Grothendieck-type inequalities in combinatorial optimization, Comm. Pure Appl. Math. 65 (2012), 9921035.

[8] G. Lancien, E. Pernecká, Approximation properties and Schauder decompositions in Lipschitz-free spaces, J. Funct. Anal. 264 (2013), 2323-2334.

[9] J. R. Lee, A. Naor, Extending Lipschitz functions via random metric partitions, Invent. Math. 160 (2005), 59-95.

[10] M. Mendel, A. Naor, Ramsey partitions and proximity data structures, J. Eur. Math. Soc. 9 (2007), 253-275.

[11] M. Mendel, A. Naor, Ultrametric skeletons, Proc. Nat. Acad. Sci. USA, 111, 48 (2013), 19251-19255.

[12] A. Naor, $L_{1}$ embeddings of the Heisenberg group and fast estimation of graph isoperimetry, Proceedings of the ICM, Hyderabad 2010, Vol. III, 1549-1575.

[13] A. Naor, An introduction to the Ribe program, Japan J. Math. 7 (2012), 167-233.

[14] P. Pansu, Difficulté d'approximation (d'après Khot, Kindler, Mossel, O'Donnell, ...), Séminaire Bourbaki, Vol. 20112012, Exposé 1045, Astérisque 352 (2013).

[15] G. Pisier, Grothendieck's theorem, past and present, Bull. AMS 49 (2012), 237-323.

[16] M. Talagrand, Regularity of Gaussian processes, Acta Mathematica 159 (1987), 99-149.

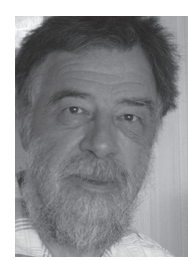

Gilles Godefroy [godefroy@math.jussieu.fr] is directeur de recherches at C.N.R.S. and a member of the Institut de Mathématiques de Jussieu (Paris). His research field is functional analysis and, in particular, the geometry of Banach spaces.

This is the English translation of the French article "De Grothendieck à Naor: une promenade dans l'analyse métrique des espaces de Banach", published in La Gazette des Mathématiciens, Société Mathématique de France (No. 151, January 2017). The author thanks J.-B. Bru and M. Gellrich Pedra for the English translation. The EMS Newsletter is thankful to La Gazette des Mathématiciens for authorisation to republish this text. 